\title{
RECPARSER: A Recursive Semantic Parsing Framework for Text-to-SQL Task
}

\author{
Yu Zeng ${ }^{1 *}$, Yan Gao ${ }^{2}$, Jiaqi Guo ${ }^{3 *}$, Bei Chen ${ }^{2}$, Qian Liu ${ }^{4 *}$ ，Jian-Guang Lou ${ }^{2}$, \\ Fei Teng ${ }^{\dagger 1}$ and Dongmei Zhang ${ }^{2}$ \\ ${ }^{1}$ Southwest Jiaotong University, Chengdu, China \\ ${ }^{2}$ Microsoft Research Asia, Beijing, China \\ ${ }^{3} \mathrm{Xi}$ ' an Jiaotong University, Xi' an, China \\ ${ }^{4}$ Beihang University, Beijing, China \\ zengyutk@my.swjtu.edu.cn, \{yan.gao, beichen, jlou, dongmeiz\}@microsoft.com, \\ jasperguo2013@stu.xjtu.edu.cn, qian.liu@buaa.edu.cn, fteng@swjtu.edu.cn
}

\begin{abstract}
Neural semantic parsers usually fail to parse long and complicated utterances into nested SQL queries, due to the large search space. In this paper, we propose a novel recursive semantic parsing framework called RECPARSER to generate the nested SQL query layer-by-layer. It decomposes the complicated nested SQL query generation problem into several progressive non-nested SQL query generation problems. Furthermore, we propose a novel Question Decomposer module to explicitly encourage RECPARSER to focus on different components of an utterance when predicting SQL queries of different layers. Experiments on the Spider dataset show that our approach is more effective compared to the previous works at predicting the nested SQL queries. In addition, we achieve an overall accuracy that is comparable with state-ofthe-art approaches.
\end{abstract}

\section{Introduction}

Text-to-SQL is one of the most important sub-tasks of semantic parsing in natural language processing (NLP). It maps natural language utterances to corresponding SQL queries. By helping non-experts to interact with ever increasing databases, the task has many important potential applications in real life, and thus receives a great deal of interest from both industry and academia [Li and Jagadish, 2016; Zhong et al., 2017; Affolter et al., 2019].

Composing nested SQL queries is a challenging problem in Text-to-SQL. Several works [Guo et al., 2019; Zhang et al., 2019b; Bogin et al., 2019; Yu et al., 2018a] have attempted to deal with this problem on the recently proposed dataset Spider [Yu et al., 2018b], which contains nested SQL queries over different databases with multiple tables. However, due to the large search space, existing neural semantic parsers do not perform well on composing nested SQL queries [Zhang et al., 2019a; Affolter et al., 2019].

\footnotetext{
${ }^{*}$ Work done during an internship at Microsoft Research.

${ }^{\dagger}$ Corresponding Author
}

\author{
Utterance: list all song names by singers age above the \\ average singer age. \\ SQL Query: SELECT song name FROM singers \\ WHERE age > (SELECT avg(age) FROM singers)
}

Figure 1: An example of the component mapping relationship between the nested SQL query and its corresponding utterance.

In this paper, we focus on the problem of composing nested SQL queries in Text-to-SQL task. Firstly, as we know the nested SQL query representation is formalized as a kind of recursive structure, thus a nested SQL query generation problem can be decomposed into several progressive non-nested SQL query generation problems by nature. Furthermore, we found that there is a strong component mapping relationship between the nested SQL query and its corresponding utterance. Figure 1 shows an example of the component mapping relationship between the nested SQL query and its corresponding utterance. We see that, for this nested SQL query, the outside layer "SELECT song_name FROM singers WHERE age >" and the inside layer "SELECT avg(age) FROM singers" correspond to different utterance components "list all song names by singers age above" and "the average singer age" respectively.

Inspired by these observations, we propose a recursive semantic parsing framework called RECPARSER to generate the nested SQL query layer-by-layer from outside to inside. Furthermore, we propose a novel Question Decomposer module to explicitly encourage RECPARSER to focus on different parts of an utterance when predicting SQL queries in different layers. RECPARSER consists of four modules: Initial Encoder, Iterative Encoder, Question Decomposer and SQL Generator. In the initialization stage, we encode the user utterance and the database schema with the Initial Encoder. Then, by recursively calling the Question Decomposer, the Iterative Encoder and the SQL Generator modules, we generate the nested SQL query layer-by-layer. Finally, we compose SQL queries of different layers into the whole SQL query when encountering a recursive termination condition. Concretely, in each recursion round, the Question Decomposer updates the utterance representation with a soft-attentionmask mechanism and a recursive-attention-divergency loss 
proposed by us for the next recursion; the Iterative Encoder contextualizes the schema representation with the updated utterance representation; the SQL Generator takes the contextualized schema representation as input and generates the nonnested SQL query of the current layer. Our SQL Generator is a simple yet effective multi-task classification model.

We argue that RECPARSER has two advantages. Firstly, in this divide-and-conquer faction, we simplify the difficulty of the nested query generation problem. Our SQL Generator only learns to generate non-nested SQL queries instead of the whole nested SQL query, thus largely reducing the search space and alleviating training difficulties. Secondly, through updating the utterance representation recursively with the soft-attention-mask mechanism and the recursive-attentiondivergency loss, RECPARSER can focus on different parts of an utterance when predicting different SQL query layers, thus minimizing the interference of irrelevant parts.

On the Spider benchmark [Yu et al., 2018b], RECPARSER achieves a state-of-the-art 39.3\% accuracy on the nested SQL query and a comparable $54.3 \%$ accuracy on the overall SQL query. When augmented with BERT [Devlin et al., 2018], RECPARSER reaches up to a state-of-the-art $46.8 \%$ accuracy on the nested SQL query and a state-of-the-art $63.1 \%$ accuracy on the overall SQL query. Our contributions are summarized as follows.

- We propose a recursive semantic parsing framework called RECPARSER to decompose a complicated nested SQL query generation problem into several progressive non-nested SQL query generation problems.

- We propose a novel Question Decomposer module with a soft-attention-mask mechanism and a recursiveattention-divergency loss to explicitly encourage RECPARSER to focus on different parts of the utterance when predicting SQL queries in different layers.

- Our approach is more effective compared to previous works at predicting nested SQL queries. In addition, we achieve an overall accuracy that is comparable with state-of-the-art approaches.

\section{Related Works}

Composing Nested SQL Query. The problem of composing nested SQL queries has been studied for decades [Androutsopoulos et al., 1995]. Most of the early proposed systems are rule-based [Popescu et al., 2003; Li and Jagadish, 2014]. Recently, with the development of advanced neural approaches and the release of the complex and cross-domain Text-to-SQL dataset Spider [Yu et al., 2018b], several neural semantic parsers [Guo et al., 2019; Zhang et al., 2019b; Bogin et al., 2019; Lee, 2019] have attempted to generate the nested SQL queries with a complicated grammar-based decoder. However, applying grammar-based decoders to general programming languages such as SQL query is very challenging [Lin et al., 2019] and difficult to generalize to other semantic parsing tasks. Also, Finegan-Dollak et al. [2018] shows that the sequence-to-tree approach is inefficient when generating complicated SQL queries from an utterance. Our work differs from them in our use of a divide-and-conquer generation procedure.
Recursive Mechanism in Semantic Parsing. Recursive mechanism has been successfully used in complicated semantic parsing tasks [Andreas et al., 2016; Rabinovich et al., 2017]. Recently, several works [Yu et al., 2018a; Lee, 2019] also employ the recursive mechanism to generate nested SQL queries in Text-to-SQL task. SyntaxSQLNet [Yu et al., 2018a] employs a SQL specific syntax treebased decoder that calls a collection of recursive modules for decoding. RCSQL [Lee, 2019] proposes a SQL clausewise decoding architecture. It recursively calls different SQL clause decoders to predict nested SQL queries. Compared with their approaches, we recursively call both encoder module and SQL generator module for each recursion, while they only call a set of SQL generator modules. In addition, we propose a novel Question Decomposer module to capture the mapping relationship between different SQL query layers and their corresponding components in utterance.

Decomposing Complicated Question. Decomposing complicated question is widely used in many semantic parsing works [Iyyer et al., 2016; Talmor and Berant, 2018; Zhang et al., 2019a]. They utilize the decompositionality of complicated questions to help question understanding. Inspired by them, we use the soft-attention-mask mechanism and the recursive-attention-divergency loss to model the decomposing process of the utterance and update the utterance representation for next recursion round. In this way, we don't need to make hard decisions and it could be learned in an end-to-end fashion without extra labeling work.

Multi-Task Learning in Text-to-SQL Task. In multi-task learning, the Text-to-SQL task is decomposed into several sub-tasks, each predicting a part of the final SQL program. Compared with sequence-to-sequence-style models, multitask learning does not require the SQL queries to be serialized and thus avoid the "ordering issue" [Xu et al., 2018]. Existing state-of-the-art multi-task learning methods [Hwang et al., 2019; He et al., 2019] have already surpassed human performance on WikiSQL [Zhong et al., 2017] dataset. However, existing methods are limited to the specific SQL sketch of WikiSQL, which only supports very simple queries. In this work, we propose a simple yet effective multi-task classification model to generate arbitrary non-nested SQL query as the SQL Generator module in our framework.

\section{Methodology}

In this section, we will describe our RECPARSER framework in detail. As described in Figure 2, RECPARSER consists of four modules: Initial Encoder, Iterative Encoder, Question Decomposer and SQL Generator. At first, we get the initial representation of utterance and database schema with the Initial Encoder. Then, by recursively calling the Iterative Encoder, the Question Decomposer, and the SQL Generator, we generate the nested SQL query layer-by-layer from outside to inside. Finally, we compose the SQL queries of different layers into the whole SQL query when encountering a recursive termination condition. 


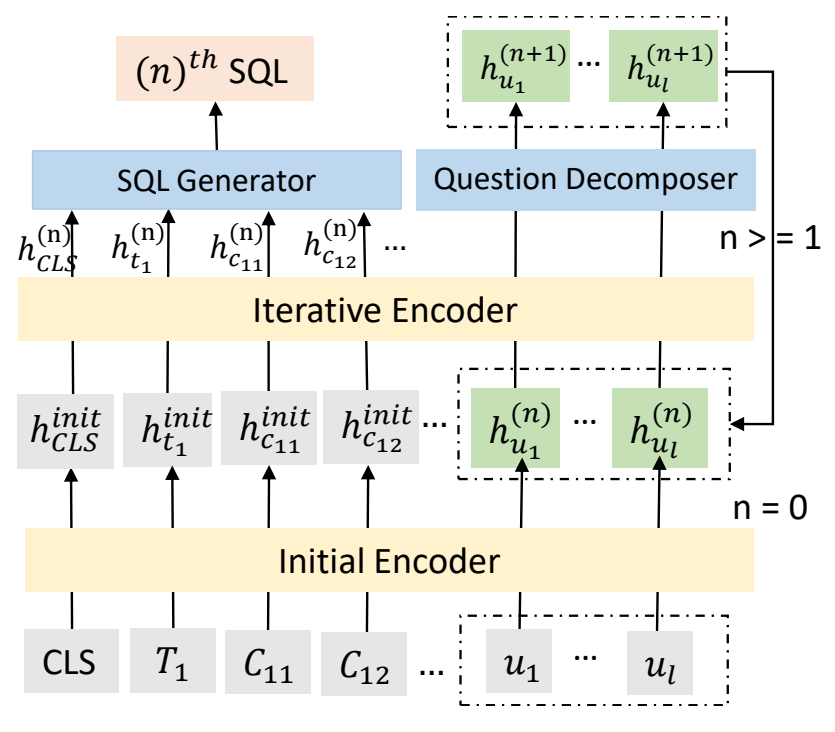

Figure 2: RECPARSER Architecture.

\subsection{Initial Encoder}

Given a user input utterance $U$ and its corresponding database schema $S$, the Initial Encoder generates the initial representation $h_{U}^{\text {init }}$ and $h_{S}^{\text {init }}$. Let $U=\left(u_{1}, . ., u_{k}, . ., u_{l}\right)$ and $S=\left\{t_{1}, c_{11}, \ldots, t_{i}, c_{i j, \ldots}\right\}$, where $u_{k}$ denotes the $k$-th utterance token, $l$ is the length of utterance, $t_{i}$ denotes the $i$-th table name in database schema, and $c_{i j}$ denotes the $j$-th column name in the $i$-th table. Following Hwang et al. [2019], we first concatenate the utterance $U$ and the database schema $S$ as a single sequence separated by token $[C L S],[T A B],[C O L]$, and $[S E P]$ as follows.

$$
[C L S],[T A B], t_{1},[C O L], c_{11} . .,[C O L], c_{i j} . .[S E P], u_{1} . . u_{l},
$$

where we put $[C L S]$ token at the beginning of the sequence to capture the contextualized representation of the whole sequence. Then we employ a Bidirectional LSTM [Hochreiter and Schmidhuber, 1997] to get the $h_{U}^{\text {init }}$ and the $h_{S}^{\text {init }}$, where $h_{U}^{\text {init }}=\left(h_{u_{1}}, . ., h_{u_{k}}, . ., h_{u_{l}}\right)$ and $h_{S}^{\text {init }}=$ $\left(h_{t_{1}} \ldots, h_{t_{i}}, h_{\text {col }_{i j}}, \ldots\right)$. Concretely, for each table and column in database schema, we use the representation of the separator $[T A B]$ and $[C O L]$ before each table and column as their corresponding representations. Moreover, we use $h_{C L S}$ denotes the representation of token $[C L S]$. By concatenating the utterance and the database schema together, we capture the relationship between the utterance and the corresponding database schema and get the contextualized representations.

\subsection{Iterative Encoder}

Let $n$ denote the $n$-th recursion round. Given $h_{U}^{(n)}, h_{C L S}^{i n i t}$ and $h_{S}^{i n i t}$ as inputs, the goal of the Iterative Encoder is to generate the database schema representation $h_{S}^{(n)}$ and the [CLS] representation $h_{C L S}^{(n)}$ of the $n$-th recursion round. Then, $h_{S}^{(n)}$ and $h_{C L S}^{(n)}$ will be used as the inputs of the SQL Generator to generate the current layer SQL query. Note that if it is the 0-th recursion round, we use $h_{U}^{i n i t}$ as $h_{U}^{0}$. Specifically, following Hwang et al. [2019], we concatenate $h_{C L S}^{\text {init }}, h_{S}^{\text {init }}$ and $h_{U}^{(n)}$

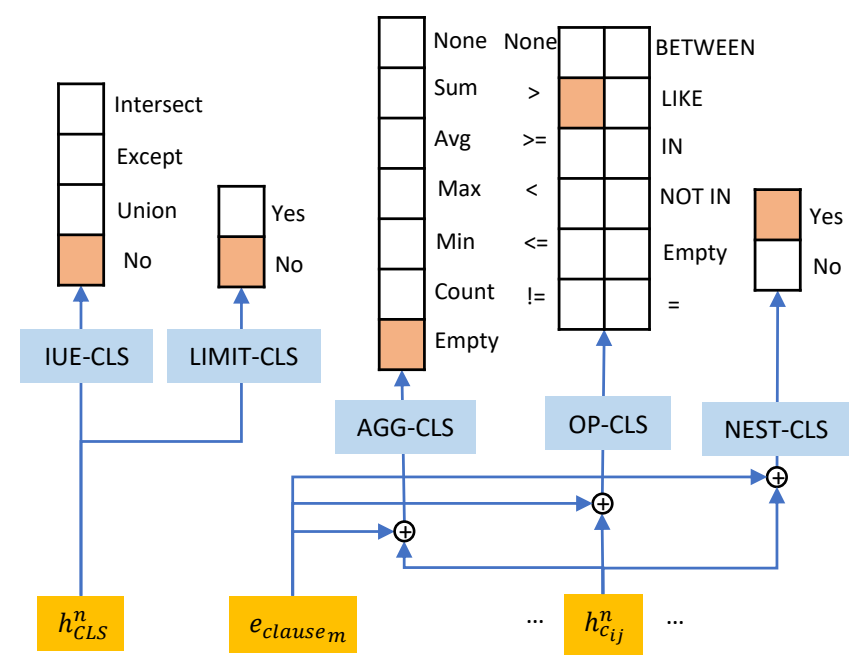

Figure 3: An overview of the SQL Generator.

in a single representation $h^{(n)}$. Then we apply self-attention mechanism [Vaswani et al., 2017] to update $h^{(n)}$ as follows.

$$
\begin{aligned}
& \alpha^{(n)}=\operatorname{softmax}\left(\frac{Q K^{T}}{\sqrt{d}}\right), \\
& h^{(n)}=\alpha^{(n)} V,
\end{aligned}
$$

where $Q=w_{q} h^{(n)}, K=w_{k} h^{(n)}, V=w_{v} h^{(n)}$ and $w_{q} \in$ $\mathbb{R}^{d \times d}, w_{k} \in \mathbb{R}^{d \times d}, w_{v} \in \mathbb{R}^{d \times d}$ are trainable parameters.

\subsection{SQL Generator}

Given the database schema representation $h_{S}^{(n)}$ and the $[C L S]$ representation $h_{C L S}^{(n)}$, the goal of the SQL Generator is to generate the non-nested SQL query at the current recursion round $n$. According to the syntactical grammar of the SQL query, we transform the non-nested SQL query generation problem to a slot-filling problem. Concretely, we design a local template that indicates the property of each column in each SQL clause (e.g., SELECT clause) and a global template that indicates the property of the SQL query.

Firstly, we generate $N \times M$ local templates where $N$ is the number of columns in the given database schema $S$ and $M$ is the number of SQL clauses. Each template has three local slots, i.e., Aggregator, Operator and Nest. Notice that the slot Nest is used to indicate whether the column in corresponding clause has nested SQL query or not. Figure 3 presents all candidates of the Aggregator, the Operator and the Nest. Notice that EMPTY means that the column is not in the corresponding SQL clause. As shown in Figure 3, they are predicted by three classifiers, i.e., AGG-CLS, OP-CLS and NES-CLS respectively. Taking the OP-CLS as an example, the probability of the operator $o p$ for $\mathrm{col}_{i j}$ in clause $_{m}$ is computed as following:

$$
\begin{aligned}
h_{\text {col }_{i j}}^{(n)} & =h_{\text {col }_{i j}}^{(n)}+e_{\text {clause }_{m}}, \\
P_{o p}^{\left(\operatorname{col}_{i j}, m\right)} & =\operatorname{softmax}\left(W_{o p} h_{\text {col }_{i j}}^{(n)}+b_{o p}\right),
\end{aligned}
$$

where $e_{\text {clause }_{m}}$ is the $m$-th clause embedding. $W_{o p} \in$ $\mathbb{R}^{n_{\text {op }} \times d}, b_{\text {op }} \in \mathbb{R}^{n_{o p}}, e_{\text {clause }_{m}} \in \mathbb{R}^{d}$ are trainable parameters 
and $n_{o p}$ is the number of operator types. The same way can be also applied to the AGG-CLS and NES-CLS to calculate $P_{a g g}^{\left(\operatorname{col}_{i j}, m\right)}$ and $P_{n e s}^{\left(\operatorname{col}_{i j}, m\right)}$. Specially, we have 7 kinds of SQL clauses (i.e., SELECT, WHERE_AND, WHERE_OR, ORDER_ASC, ORDER_DES, GROUP, and HAVING), in which the original WHERE clause is divided into WHERE_AND and WHERE_OR to distinguish two different connectors (i.e., AND and OR) in the WHERE clause. Similarly, the ORDER clause is also divided into ORDER_ASC and ORDER_DES to distinguish two different sort types (i.e., DES and ASC).

Next, we generate one global template which has two global slots, i.e., Limit and IUE. Limit indicates if the SQL query contains a keyword LIMIT. IUE indicates whether the SQL query contains structures such as Intersect, Union, Except or not. We use another two classifiers, i.e., LIMIT-CLS and IUE-CLS to predict them respectively. In IUE-CLS, the probability of the candidate iue is calculated as follows.

$$
P_{i u e}=\operatorname{softmax}\left(W_{i} h_{C L S}^{(n)}+b_{i}\right),
$$

where $W_{i} \in \mathbb{R}^{n_{i} \times d}, b_{i} \in \mathbb{R}^{n_{i}}$ are trainable parameters, $n_{i}$ is the number of IUE types. $P_{\text {limit }}$ is calculated in the same way by LIMIT-CLS. After filling all the slots of all the templates, we use a heuristic method to compose these templates to a SQL query according to the SQL grammar.

At last, after predicting all the other clauses, similar to [Lee, 2019], we use a heuristics to generate the FROM clause of the current SQL query layer. We first collect all the columns that appear in the predicted SQL, and then we JOIN tables that include these predicted columns.

\subsection{Question Decomposer}

The goal of the Question Decomposer is to update the utterance representation for the next recursion round. Intuitively, we hope that RECPARSER could learn to decompose the utterance layer-by-layer and focus on different components of an utterance when predicting SQL queries of different layers. We achieve this target by two simple yet effective methods: (1) Soft Attention Mask. Inspired by Kim et al. [2018] which leverages a soft attention mask mechanism to do feature selection, we propose a novel method to soft mask the parts of utterance that has already been focused by previous recursion rounds. (2) Recursive Attention Divergence Loss. Inspired by Wei et al. [2019] which uses JS divergence loss to minimize the attention divergence in code generation task, we propose a novel regularization loss to encourage the model to maximize the attention divergence between the adjacent recursion rounds. In this way, we explicitly encourage RECPARSER to focus on different components of an utterance when predicting different SQL query layers, thus minimizing the interference of irrelevant components of the utterance.

\section{Soft Attention Mask}

Let $\alpha_{u_{i}}^{(n)}$ denotes the attention weight of token $[C L S]$ on $i$ th token of utterance $U$, which is produced by Equation 2 . Intuitively, to help the model focus on different components of an utterance in the next recursion, we want to mask the component that has been focused on the current recursion. Instead of directly masking the tokens of the utterance, we generate the utterance representation of next recursion by a soft-attention-mask method as follows.

$$
\begin{aligned}
E_{\text {mask }}^{(n)} & =\alpha_{u_{i}}^{(n)} W_{\text {mask }}, \\
h_{u_{i}}^{(n+1)} & =W_{U} h_{u_{i}}^{(n)}+W_{m} E_{\text {mask }}^{(n)},
\end{aligned}
$$

where $E_{\text {mask }}^{(n)} \in \mathbb{R}^{d}$ is the soft mask embedding, $W_{\text {mask }} \in$ $\mathbb{R}^{d}, W_{U} \in \mathbb{R}^{d \times d}, W_{m} \in \mathbb{R}^{d \times d}$ are trainable parameters.

\section{Recursive Attention Divergence Loss}

To encourage the model to focus on different components of an utterance in each recursion round, we design the Recursive Attention Divergence Loss $\mathcal{L}_{\text {div }}$ to regularize the attention divergence between the attention weights of the adjacent recursion rounds.

Let $\alpha_{U}^{(n)}$ and $\alpha_{U}^{(n-1)}$ denote the attention weights of the adjacent recursion rounds for a same utterance. We apply the negative Jensen-Shannon divergence [Fuglede and Topsoe, 2004], a symmetric measurement of similarity between two probability distributions, to maximize the distance between these two attention weights. Let $P$ denotes the $\alpha_{U}^{(n)}$ and $Q$ denotes the $\alpha_{U}^{(n-1)}$ :

$$
\mathcal{L}_{d i v}=-\frac{1}{2}\left(D_{K L}\left(P \| \frac{P+Q}{2}\right)+D_{K L}\left(Q \| \frac{P+Q}{2}\right)\right),
$$

where $D_{K L}$ is the Kullback-Leibler divergence, defined as $D_{K L}(p \| q)=\sum_{x} p(x) \log \left(\frac{p(x)}{q(x)}\right)$ which measures how one probability distribution $q$ diverges from another probability distribution $p$.

\subsection{Composing the Final SQL Query}

The final SQL query is obtained through backtracking. The SQL Generator will return a SQL query segment after the end of the recursive calling. The recursive termination condition is that the IUE slot and all NEST slots are NO. Specifically, if the slot NEST is YES, we return a SQL query with a placeholder token [SUBQUERY] after its corresponding column that is in its clause (e.g., SELECT name FROM singers WHERE age $>$ [SUBQUERY] and sex $=$ 'male'). Similarly, if the IUE slot is not NO, we put corresponding placeholder token after the returned SQL query result (e.g., SELECT name FROM singers [EXCEPT]). Finally, we construct the final SQL by replacing the placeholder token with the returned SQL query segment.

\subsection{Loss Function}

The loss function of RECPARSER is defined as follows.

$$
\begin{gathered}
\mathcal{L}=\lambda_{A G G} \mathcal{L}_{A G G}+\lambda_{O P} \mathcal{L}_{O P}+\lambda_{N E S} \mathcal{L}_{N E S} \\
+\lambda_{I U E} \mathcal{L}_{I U E}+\lambda_{L I M I T} \mathcal{L}_{L I M I T}+\lambda_{d i v} \mathcal{L}_{d i v}
\end{gathered}
$$

where $\mathcal{L}_{A G G}, \mathcal{L}_{O P}, \mathcal{L}_{N E S}, \mathcal{L}_{I U E}, \mathcal{L}_{L I M I T}$ are the loss functions of AGG-CLS, OP-CLS, NES-CLS, IUE-CLS and LIMIT-CLS in the SQL Generator respectively. All loss functions of the classifiers are cross-entropy loss. $\mathcal{L}_{\text {div }}$ is the $\mathrm{Re}-$ cursive Attention Divergence Loss described in Equation 6. $\lambda_{A G G}, \lambda_{O P}, \lambda_{N E S}, \lambda_{I U E}, \lambda_{L I M I T}, \lambda_{d i v}$ are loss weights and all of them are hyper parameters. 


\begin{tabular}{ccc}
\hline Approach & Accuracy & Accuracy (BERT) \\
\hline SyntaxSQLNet & 0 & - \\
Global-GNN & $33.0 \%$ & - \\
EditSQL & - & $40.4 \%$ \\
IRNET & $34.2 \%$ & $41.8 \%$ \\
RECPARSER(no DB) & $\mathbf{3 8 . 0 \%}$ & $\mathbf{4 6 . 8 \%}$ \\
RECPARSER & $\mathbf{3 9 . 3 \%}$ & $\mathbf{4 6 . 8 \%}$
\end{tabular}

Table 1: Nested SQL Exact Matching accuracy on Spider development set. "no DB" means the database content is not used; "BERT" means the results are obtained with BERT enhanced approaches and "-" means the methods are not proposed in corresponding settings, e.g., with or without BERT.

\section{Experiments}

In this section, we evaluate the effectiveness of RECPARSER on both nested SQL queries and all SQL queries by comparing it to state-of-the-art approaches and ablating several design choices in RECPARSER to understand their contributions.

\subsection{Experiment Setup}

Dataset. We conduct our experiments on Spider [Yu et al., 2018b], a large-scale, human-annotated and crossdomain Text-to-SQL benchmark, which contains 7,000/1,034 question-SQL query pairs in train and development set ${ }^{1}$. We using SQL Exact Matching and Component Matching accuracy metrics proposed by Yu et al. [2018b] to evaluate RECPARSER and other approaches.

Baselines. We use the following methods for comparison evaluation: SyntaxSQLNet [Yu et al., 2018a], RCSQL [Lee, 2019], Global GNN [Bogin et al., 2019], EditSQL [Zhang et al., 2019b], and IRNET [Guo et al., 2019]. All of them employ an encoder-decoder architecture, but with different design choices: SyntaxSQLNet is a sequence-to-set model with a SQL specific syntax tree-based decoder; RCSQL employs a SQL clause-wise decoding network with recursive mechanism; Global GNN reasons over the DB structure and questions to make global decision; EditSQL proposes an editing mechanism-based network; and IRNET uses schema linking information and predicts an intermediate representation instead of the SQL query. Note that SyntaxSQLNet, RCSQL, EditSQL and IRNet do not use the database content, while Global GNN and RECPARSER use the database content.

Implementations. We implement RECPARSER with PyTorch. The dropout rate is 0.2. We use Adam with 1e-3 learning rate for optimization. Batch size is 64. Word embeddings are initialized with Glove. The dimensions of word embedding, type embedding and hidden vectors are set to 300. $\lambda_{A G G}, \lambda_{O P}, \lambda_{N E S}, \lambda_{I U E}, \lambda_{L I M I T}, \lambda_{d i v}$ in Equation 7 are set as $1,1,1,0.1,0.1$, and 0.2 respectively. We use the database content to find the column whose value is exactly mentioned in the utterance and put a special token [VALUE] in front of that column.

\footnotetext{
${ }^{1}$ We don't use the test set of Spider, since the test set is blind to us and we can't obtain the nested SQL query of the test set to evaluate the effectiveness of our approach.
}

\begin{tabular}{ll}
\hline Approach & Accuracy \\
\hline SyntaxSQLNet & $24.8 \%$ \\
RCSQL & $28.5 \%$ \\
Global-GNN & $52.7 \%$ \\
IRNET & $53.2 \%$ \\
RECPARSER & $\mathbf{5 4 . 3 \%}$ \\
\hline Edit-SQL(BERT) & $57.6 \%$ \\
RECPARSER(BERT, no DB) & $60.5 \%$ \\
IRNET(BERT) & $61.9 \%$ \\
RECPARSER(BERT) & $\mathbf{6 3 . 1 \%}$ \\
\hline
\end{tabular}

Table 2: SQL Exact Matching accuracy on Spider development set

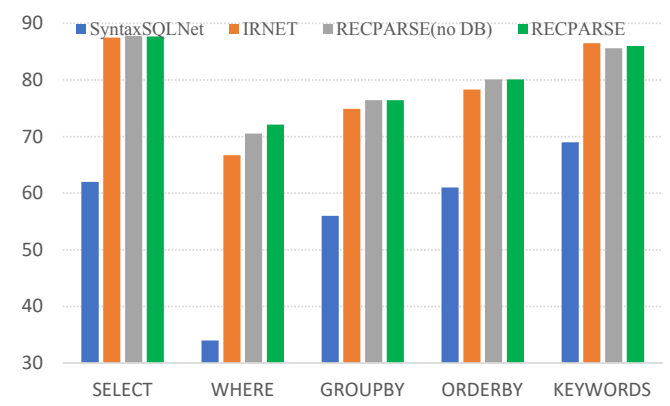

Figure 4: F1 scores of Component Matching accuracy of SyntaxSQLNet, IRNET, RECPARSER(no DB) and RECPARSER on Spider development set. All methods are augmented with BERT.

BERT. Language model pre-training has shown to be effective for learning universal language representations. To further study the effectiveness of our approach, we leverage BERT [Devlin et al., 2018] to encode question and database schema to replace the bi-directional LSTM in the Initial Encoder. Our approach with BERT-base is denoted as RECPARSER(BERT).

\subsection{Model Comparison}

Table 1 presents the nested SQL Exact Matching accuracy of RECPARSER and various baselines on the development set. The result shows that RECPARSER clearly outperforms all the baselines with or without DB content used on the nested SQL query accuracy. Comparing to the methods that use various techniques to predict nested SQL query, such as complicated intermediate representation (e.g., IRNet) and other grammar-based decoders (e.g., EditSQL and Global-GNN), RECPARSER achieves a prominent improvement by employing a novel recursive generation framework. For example, it obtains $5.0 \%$ absolute improvement than the state-of-the-art method IRNET when both of them incorporate with BERT.

Next, we evaluate the overall SQL Exact Matching accuracy of RECPARSER and various baselines on the development set of Spider. As shown in Table 2, RECPARSER(BERT, no DB) achieves a $60.5 \%$ Exact Matching accuracy without using any rule-based schema linking techniques or intermediate representation that are used in IRNet(BERT). It demonstrates that RECPARSER(BERT, no DB) obtains a comparable overall accuracy than the state-of-the-art approaches at the same setting with min- 


\begin{tabular}{|c|c|c|c|}
\hline \multirow{2}{*}{ Case 1} & $\begin{array}{l}\text { With } \\
\text { QD }\end{array}$ & $\begin{array}{l}\text { Round 1: What is the number of cars with a greater accelerate than the accelerate of car with the most horsepower? } \\
\text { Round 2: What is the number of cars with a greater accelerate than the accelerate of car with the most horsepower? } \\
\text { Result: SELECT count }(*) \text { FROM cars_data WHERE accelerate > (SELECT accelerate FROM cars_data ORDER BY horsepower DESC LIMIT } 1 \text { ) }\end{array}$ & $\sqrt{ }$ \\
\hline & $\begin{array}{c}\text { Without } \\
\text { QD }\end{array}$ & $\begin{array}{l}\text { Round 1: What is the number of cars with a greater accelerate than the accelerate of with the most } \\
\text { than the accelerate of car with the most horsepower? } \\
\text { Round 2: What is the number of cars with a }\end{array}$ & $x$ \\
\hline \multirow[t]{2}{*}{ Case 2} & $\begin{array}{l}\text { With } \\
\text { QD }\end{array}$ & $\begin{array}{l}\text { Round 1: Find the names museums which have more staff than the minimum staff number of all museums opened after } 2010 . \\
\text { Round 2: Find the names of museums which have more staff the minimum staff number of all museums } \\
\text { Result: SELECT name FROM museum WHERE num_of_staff > (SELECT min(num_of_staff) FROM museum WHERE open_year }>2010 \text { ) }\end{array}$ & $\sqrt{ }$ \\
\hline & $\begin{array}{l}\text { Without } \\
\text { QD }\end{array}$ & $\begin{array}{l}\text { Round 1: Find the names museums which have more staff than the minimum staff } \\
\text { Round 2: Find the names all museums opened after } 2010 . \\
\text { Result: SELECT name FROM museum Which have more } \\
\text { than the minimum staff number of all museums opened } 2010 .\end{array}$ & $x$ \\
\hline
\end{tabular}

Figure 5: Two examples where Question Decomposer (QD) for RECPARSER leads to correct prediction. In the first example, RECPARSER without QD selects the wrong column in the outside SQL layer which belongs to the inner SQL layer. In the second example, RECPARSER without QD selects the wrong column in the inner SQL layer which belongs to the outside SQL layer. Color intensity in red reflects the attention weight, and the tokens in black have negligibly small weights.

\begin{tabular}{ll}
\hline Base-framework & $57.4 \%$ \\
\hline + QD (- RAD loss) & $58.5 \%$ \\
+ QD & $60.8 \%$ \\
+ QD + DB & $63.1 \%$ \\
\hline
\end{tabular}

Table 3: Ablation study results. Base-framework doesn't use the Question Decomposer (QD) and the database content (DB). 'QD ($\mathrm{RAD}$ loss)' means that the QD does not use the recursive-attentiondivergency loss.

imum efforts. When incorporating the database content, RECPARSER(BERT) gets a $63.1 \%$ Exact Matching accuracy and obtains a $1.2 \%$ absolute improvement over state-ofthe-art method. It demonstrates the effectiveness of RECPARSER(BERT).

To further study the performance of RECPARSER in detail, following Yu et al. [2018a], we measure the average F1 score on different SQL query components on the development set. Here, we take BERT enhanced methods as an example. As shown in Figure 4, RECPARSER outperforms SyntaxSQLNet and IRNET on all components except KEYWORDS. Importantly, because of the good performance of RECPARSER on nested SQL query which belongs to the WHERE clause, there is a $3.4 \%$ absolute improvement compared to IRNET in WHERE clause. When incorporating with database content, the improvement is boosted to $5.4 \%$.

\subsection{Ablation Study}

We conduct ablation study on RECPARSER(BERT) to analyze the contribution of each design choice. In detail, we first evaluate a base framework that does not use the Question Decomposer (QD) and the database content (DB). Then, we gradually apply each component to the base model. The ablation study is conducted on the development set. Table 3 shows the results of ablation study.

Firstly, it is clear that our base model significantly outperforms the well designed complicated architecture methods (e.g., Global-GNN and SyntaxSQLNet) by using the recursive framework with the simple yet effective multi-task SQL Generator module.

Secondly, using the Question Decomposer without the recursive-attention-divergency loss improves the perfor- mance of RECPARSER about $1.1 \%$. It demonstrates the effectiveness of using the soft-mask-attention to update the utterance representation.

Thirdly, adding the recursive-attention-divergency loss further brings a $2.3 \%$ improvement. It demonstrates the effectiveness of our loss function. We observe that for those nested SQL queries, the base framework has the problem that tends to put the columns of the outside layer to the inside layer. The number of examples suffering from this problem decreases by 53\% when using the Question Decomposer. It means that the Question Decomposer helps to minimize the interference between different SQL layers. At last, using the database content helps to recognize the value mentioned in the utterance, which brings a $2.3 \%$ improvement of performance.

\subsection{Qualitative Analysis}

Here we conduct a qualitative analysis on the effectiveness of the Question Decomposer (QD). Firstly, Figure 5 visualizes the attention weights $\alpha_{U}$ from Equation 2 in different rounds of the two examples, in which color intensity reflects the attention weight. As we can see, when incorporating the QD module, RECPARSER is much easier to focus on different components of the utterance between different rounds than removing the QD module. Secondly, according to the prediction results of the two examples, when removing the QD module, RECPARSER tends to be confused about the correct layer position of the selected column. When incorporating with the QD module, both of the two examples get the correct SQL queries. It demonstrates that the QD module help RECPARSER to minimize the interference between different utterance components that correspond to different SQL query layers.

\section{Conclusion}

In this paper, we propose a novel recursive semantic parsing framework called RECPARSER to decompose the complicated nested SQL generation problem into several progressive non-nested SQL query generation problems. Experiments on Spider dataset show that our approach is more effective compared to previous work at predicting the nested SQL queries. In addition, we achieve a comparable overall accuracy to state-of-the-art approaches. 


\section{References}

[Affolter et al., 2019] Katrin Affolter, Kurt Stockinger, and Abraham Bernstein. A comparative survey of recent natural language interfaces for databases. The VLDB Journal, 28:793 - 819, 2019.

[Andreas et al., 2016] Jacob Andreas, Marcus Rohrbach, Trevor Darrell, and Dan Klein. Learning to compose neural networks for question answering. In $N A A C L$, pages 1545-1554, San Diego, California, June 2016. Association for Computational Linguistics.

[Androutsopoulos et al., 1995] Ion Androutsopoulos, Graeme D Ritchie, and Peter Thanisch. Natural language interfaces to databases-an introduction. Natural language engineering, 1(1):29-81, 1995.

[Bogin et al., 2019] Ben Bogin, Matt Gardner, and Jonathan Berant. Global reasoning over database structures for text-to-sql parsing. In EMNLP/IJCNLP, 2019.

[Devlin et al., 2018] Jacob Devlin, Ming-Wei Chang, Kenton Lee, and Kristina Toutanova. Bert: Pre-training of deep bidirectional transformers for language understanding. arXiv preprint arXiv:1810.04805, 2018.

[Finegan-Dollak et al., 2018] Catherine Finegan-Dollak, Jonathan K. Kummerfeld, Li Zhang, Karthik Ramanathan, Sesh Sadasivam, Rui Zhang, and Dragomir Radev. Improving text-to-SQL evaluation methodology. In $A C L$, pages 351-360, Melbourne, Australia, July 2018. Association for Computational Linguistics.

[Fuglede and Topsoe, 2004] Bent Fuglede and Flemming Topsoe. Jensen-shannon divergence and hilbert space embedding. In International Symposium onInformation Theory, 2004. ISIT 2004. Proceedings., page 31. IEEE, 2004.

[Guo et al., 2019] Jiaqi Guo, Zecheng Zhan, Yan Gao, Yan Xiao, Jian-Guang Lou, Ting Liu, and Dongmei Zhang. Towards complex text-to-sql in cross-domain database with intermediate representation. In $A C L, 2019$.

[He et al., 2019] Pengcheng He, Yi Mao, Kaushik Chakrabarti, and Weizhu Chen. X-sql: reinforce schema representation with context. arXiv preprint arXiv:1908.08113, 2019.

[Hochreiter and Schmidhuber, 1997] Sepp Hochreiter and Jürgen Schmidhuber. Long short-term memory. Neural computation, 9(8):1735-1780, 1997.

[Hwang et al., 2019] Wonseok Hwang, Jinyeung Yim, Seunghyun Park, and Minjoon Seo. A comprehensive exploration on wikisql with table-aware word contextualization. arXiv preprint arXiv:1902.01069, 2019.

[Iyyer et al., 2016] Mohit Iyyer, Wen tau Yih, and Ming-Wei Chang. Answering complicated question intents expressed in decomposed question sequences. ArXiv, abs/1611.01242, 2016.

[Kim et al., 2018] Wonsik Kim, Bhavya Goyal, Kunal Chawla, Jungmin Lee, and Keunjoo Kwon. Attention-based ensemble for deep metric learning. In ECCV, pages 736-751, 2018.

[Lee, 2019] Dongjun Lee. Clause-wise and recursive decoding for complex and cross-domain text-to-sql generation. In EMNLP/IJCNLP, pages 6047-6053, 2019.

[Li and Jagadish, 2014] Fei Li and HV Jagadish. Constructing an interactive natural language interface for relational databases. Proceedings of the VLDB Endowment, 8(1):73-84, 2014.

[Li and Jagadish, 2016] Fei Li and H. V. Jagadish. Understanding natural language queries over relational databases. SIGMOD Record, 45:6-13, 2016.
[Lin et al., 2019] Kevin Lin, Ben Bogin, Mark Neumann, Jonathan Berant, and Matt Gardner. Grammar-based neural text-to-sql generation. arXiv preprint arXiv:1905.13326, 2019.

[Popescu et al., 2003] Ana-Maria Popescu, Oren Etzioni, and Henry Kautz. Towards a theory of natural language interfaces to databases. In Proceedings of the 8th international conference on Intelligent user interfaces, pages 149-157. ACM, 2003.

[Rabinovich et al., 2017] Maxim Rabinovich, Mitchell Stern, and Dan Klein. Abstract syntax networks for code generation and semantic parsing. In $A C L$, pages 1139-1149, Vancouver, Canada, July 2017. Association for Computational Linguistics.

[Talmor and Berant, 2018] Alon Talmor and Jonathan Berant. The web as a knowledge-base for answering complex questions. In NAACL, pages 641-651, New Orleans, Louisiana, June 2018. Association for Computational Linguistics.

[Vaswani et al., 2017] Ashish Vaswani, Noam Shazeer, Niki Parmar, Jakob Uszkoreit, Llion Jones, Aidan N Gomez, Łukasz Kaiser, and Illia Polosukhin. Attention is all you need. In NIPS, pages 5998-6008, 2017.

[Wei et al., 2019] Bolin Wei, Ge Li, Xin Xia, Zhiyi Fu, and Zhi Jin. Code generation as a dual task of code summarization. In NIPS, pages 6559-6569, 2019.

[Xu et al., 2018] Xiaojun Xu, Chang Liu, and Dawn Xiaodong Song. Sqlnet: Generating structured queries from natural language without reinforcement learning. ArXiv, abs/1711.04436, 2018.

[Yu et al., 2018a] Tao Yu, Michihiro Yasunaga, Kai Yang, Rui Zhang, Dongxu Wang, Zifan Li, and Dragomir Radev. SyntaxSQLNet: Syntax tree networks for complex and cross-domain text-to-SQL task. In EMNLP, pages 1653-1663, Brussels, Belgium, October-November 2018. Association for Computational Linguistics.

[Yu et al., 2018b] Tao Yu, Rui Zhang, Kai Yang, Michihiro Yasunaga, Dongxu Wang, Zifan Li, James Ma, Irene Li, Qingning Yao, Shanelle Roman, Zilin Zhang, and Dragomir R. Radev. Spider: A large-scale human-labeled dataset for complex and crossdomain semantic parsing and text-to-sql task. In EMNLP, 2018.

[Zhang et al., 2019a] Haoyu Zhang, Jingjing Cai, Jianjun $\mathrm{Xu}$, and Ji Wang. Complex question decomposition for semantic parsing. In $A C L, 2019$.

[Zhang et al., 2019b] Rui Zhang, Tao Yu, He Yang Er, Sungrok Shim, Eric Xue, Xi Victoria Lin, Tianze Shi, Caiming Xiong, Richard Socher, and Dragomir R. Radev. Editing-based sql query generation for cross-domain context-dependent questions. In EMNLP/IJCNLP, 2019.

[Zhong et al., 2017] Victor Zhong, Caiming Xiong, and Richard Socher. Seq2sql: Generating structured queries from natural language using reinforcement learning. CoRR, abs/1709.00103, 2017. 